\title{
GESCHRIEBENE UND GESPROCHENE SPRACHE DIE UNTERSUCHUNG EINES MUNDLICHEN UND SCHRIFTLICHEN TEXTES
}

\author{
Selma Akol - Hikmet Asutay
}

\begin{abstract}
Die geschriebene- und die gesprochene Sprache beinhalten natürlich Besonderheiten, wenn man den Fall aus der Sicht von der Sprachwissenschaft, bzw aus der Grammatik sieht. In dieser Arbeit werden die Merkmale von gesprochener und geschriebener Sprache herausgearbeitet, wobei eine Fülle von Beispielen das Verständnis erleichtern soll. Und dann handelt es sich um eine Untersuchung, die über eine Handlung vom mündlichen und schriftlichen Erklären und der Vergleich Schriftsprache- gesprochene Sprache ist. Zur sprachwissenschaftlich-analytischen Beispiel wurde ist hier das Thema "wie benutze ich "antolin" im Internet für den Unterricht?". Die zufällige Person ist ein zehnjähriger Türkisch-stämmiger Schüler einer vierten Klasse in einer Grundschule in Lengerich (NRW).
\end{abstract}

Schlüsselwort: Sprache, mündlicher text, sprachlicher text, schreiben, sprechen.

\section{KONUŞMA DÍLİ ILE YAZI DILI SÖZLÜ VE YAZILI BİR METINN İNCELEMESİ}

\section{$\ddot{O}_{z e t}$}

Dilbilim ve özellikle dilbilgisi açısından bakıldığında konuşma dili ile yazı dili arasında doğal olarak büyük ayrımlar olduğu görülür. Bu çalışmada ele alınan örneklemeler çerçevesinde bu farkllliklar ortaya konarak anlamin nasıl daha kolaylaşttrılabileceği konusu dile getirilmeye çallşılacak, örnekler doğrultusunda konuşma ve yazma eylemleri irdelenerek konuşma dili ile yazı dili arasindaki farklıliklar çözümleyici olarak incelenecektir. Bu anlamdaki dilbilimsel-çözümlemeci karşılaştırma örneğimiz için konu ders için internette ,, antolin “ olacak, rastlantısal olarak seçilmiş olan denek ise, Türk asıllı on yaşındaki Lengerich'te ilkokul dördüncü slnıf ögrencisi olacaktır.

Anahtar kelimeler: dil, konuşma metni, yazll metin, yazma, konuşma. 


\section{Einleitung}

Gesprochene und geschriebene Sprache umgibt uns jeden Tag. Wir kommunizieren mit Freunden, der Familie und am Arbeitsplatz, wir lesen Bücher, Hinweisschilder oder Kochrezepte, wir telefonieren oder schreiben kurze Mitteilungen. Dass wir uns bei alldem unterschiedlicher Ausformungen von Sprache bedienen, ist uns zumeist nicht bewusst. Gesprochene und geschriebene Sprache sind zwei mögliche Formen von kommunikativen Praktiken.

In dieser Arbeit werden die Merkmale von gesprochener und geschriebener Sprache herausgearbeitet, wobei eine Fülle von Beispielen das Verständnis erleichtern soll. Und dann handelt es sich um eine Untersuchung, die über eine Handlung vom mündlichen und schriftlichen Erklären und der Vergleich Schriftsprache- gesprochene Sprache ist. Das Thema ist "wie benutze ich "antolin" im Internet für den Unterricht?". Die Person ist ein zehnjähriger türkisch-stämmiger Schüler einer vierten Klasse in einer Grundschule in Lengerich (NRW).

\section{Gesprochene und geschriebene Sprache}

In einer Sprache, die eine Schrift besitzt, kann man sich mündlich oder schriftlich verständigen. Sprache ist einerseits gesprochene Sprache. Als Lautsprache ist sie ein akustisches Phänomen. Sie reicht nur so weit, wie die Stimme tragt, und ist nur so lange vorhanden, wie die Schallwelle Dauer hat. Andererseits ist die Sprache geschriebene Sprache und damit ein visuelles Phänomen. Mithilfe der Schrift ist es möglich, der Sprache Dauer zu verleihen und sie räumlich zu verbreiten. Zwischen beiden Materialisierungen von Sprache, der gesprochenen und geschriebenen, bestehen bedeutsame Unterschiede. Der normale Gebrauch der gesprochenen Sprache ist situationsbezogen. Die Sprechsituation ist dieselbe Situation für den Sprecher wie den Hörer. Dies hat weit reichende Folgen für den Sprachgebrauch. So können sich Sprecher und Hörer ohne viele Worte auf die Gegenstände beziehen, über die sie sprechen (Duden, Die Grammatik; Dudenverlag, 2005. S:61).

Auch mit geschriebener Sprache wird kommuniziert, tritt der Schreiber mit seinen Lesern oder “Adressaten” in Beziehung. Diese Beziehung ist aber indirekt. Häufig weiß man gar nicht, wer einen Text geschrieben hat oder wer ihn liest, der Leser ist typischerweise gerade nicht in der Schreibsituation anwesend. Wäre er anwesend, so würde er angesprochen, nicht angeschrieben (Duden, Die Grammatik; Dudenverlag, 2005. S:62).

\subsection{Zur Unterscheidung gesprochener und geschriebener Sprache}

Gesprochene Sprache unterscheidet sich von geschriebener durch eine Reihe von 
kommunikativen und strukturellen Eigenschaften. Sie können wie folgt zusammengefasst werden:

„Gesprochene Sprache ist flüchtig, unsichtbar, linear (sukzessive), irreversibel, kontextabhängig, meist dialogisch, ist auf raum-zeitliche Kopräsenz der Interaktanten angewiesen.

Geschriebene Sprache ist dauerhaft, sichtbar, räumlich (simultan), reversibel, kontextunabhängiger, meist monologisch, ermöglicht zeitliche und räumliche Trennung der Interaktanten (Springer 2000)“.

\section{Was sind prototypische Merkmale?}

Die Definition prototypischer Merkmale ist also weitgehend subjektiv und kann darum nur als Hilfskonstruktion dienen. So wird in der gesprochenen Sprache eine Situation von Angesicht zu Angesicht (face-to-face) als prototypisch empfunden, z.B. Arbeitsgespräch, Unterricht oder Kaffeeklatsch. Nicht prototypisch hingegen wären Telefongespräche, Mitteilungen auf dem Anrufbeantworter, CDs oder Ähnliches. In der geschriebenen Sprache wäre dagegen ein sprachlich elaborierter Text prototypisch, beispielsweise ein Roman, Gedicht, Werbetext oder auch eine Gebrauchsanweisung. Als nicht prototypisch würden hier beispielsweise Grußkarten, Notizzettel oder Tafelanschriebe gelten.

Die gesprochene Sprache ist phylogenetisch und ontogenetisch primär. Das heißt sowohl in der Menschheitsentwicklung als auch in der individuellen Entwicklung jedes einzelnen Menschen erfolgt die gesprochene Sprache vor der geschriebenen Sprache. Es gibt viele Kulturen ohne Schrift, aber keine ohne Sprache. Es gibt nicht alphabetisierte Menschen, aber keine, die nicht sprechen können. Abgesehen von Gehörlosen, die in diesem Zusammenhang eine Ausnahme darstellen. Zu bedenken ist hierbei auch, dass geschriebene Sprache im Gegensatz zur gesprochenen von Kindern nicht nebenbei erlernt wird, sondern nur durch mühsame Arbeit erlangt werden kann. Grundsätzlich lässt sich feststellen, dass jede Schriftsprache zwei wesentliche Formen des Ausdrucks hat. Zum einen die phonetisch realisierte Form, nämlich die gesprochene Sprache. Zum andern die graphisch realisierte Form, das heißt die geschriebene Sprache (Metzler 2000:246).

\subsection{Prototypische Merkmale beider Sprachformen}

Beide Formen von Sprache, die gesprochene und die geschriebene besitzen spezifische, prototypische Eigenschaften, die hier im Folgenden nach Dürscheid (2004), Fiehler, Barden, Elstermann und Kraft (2004) und Metzler (2000) vereinfacht zusammengefasst sind, ohne dabei einen Anspruch auf Vollständigkeit zu erheben: 
- Gesprochene Sprache ist flüchtig und nicht wiederholbar. Geschriebene Sprache kann archiviert werden und ist immer in der gleichen Form rückholbar. Das verdeutlicht besonders drastisch ein Vergleich zwischen der Entstehung von Gerüchten, die steten Wandlungen unterworfen sind und Steininschriften früher Kulturen, die noch heute den gleichen Inhalt wiedergeben. Als Ausnahmen, nicht prototypische Formen gelten hier Tonbandaufzeichnungen und Geschriebenes, das vorgelesen wird.

- Gesprochene Sprache unterliegt den Bedingungen von Zeit und Raum und damit einer Kommunikationssituation von Angesicht zu Angesicht. Geschriebene Sprache ist nicht an eine gemeinsame Äußerungssituation gebunden. Bei der gesprochenen Sprache verläuft das Senden (Sprechen) und das Empfangen (Hören) synchron; sie sind zeitlich gekoppelt. Der Hörer hat zudem die Möglichkeit dem Sprecher ins Wort fallen. Ausnahmen bilden hier Aufzeichnungen jeder Art oder Radiobeiträge. Bei der geschriebenen Sprache verläuft die Kommunikation asynchron, sie ist zeitlich entkoppelt und eine Intervention ist damit nicht möglich. Als Ausnahme wären hier Chats zu nennen.

- Die gesprochene Sprache verwendet deiktische Ausdrücke. Sprecher und Hörer verfügen hier nach Bühler über ein gemeinsames Zeigfeld, was erst durch die zeitliche und räumliche Koppelung beider ermöglicht wird. Beispielsweise haben hier Begriffe wie: hier, da rechts, hinten, gestern, nächstes Jahr eine konkrete, für den Hörer verständliche Bedeutung. Die geschriebene Sprache muss sich dagegen sehr klar und präzise ausdrücken, weil bei Schreiber und Leser kein gemeinsames Zeigfeld vorhanden ist. Der Text muss also losgelöst von der Produktionssituation verstehbar sein und der Schreiber muss seine Äußerungen explizieren. Die gesprochene Aussage „Gestern sah ich den neuen Airbus“, müsste in der geschriebenen Sprache „Am 9. Mai sah ich den Airbus 380“ heißen.

- Bei der gesprochenen Sprache werden weitere Informationsträger, wie Mimik, Gestik und Intonation verwendet. Ein und derselbe Satz kann so bei unterschiedlicher Intonation verschiedene Bedeutungen bekommen, in ironischer Form sogar das Gegenteil bedeuten. Die geschriebene Sprache muss ohne diese zusätzlichen Informationsträger auskommen. Emotionen müssen hier mit rein sprachlichen Mitteln zum Ausdruck kommen.

- Die gesprochene Sprache ist nicht auf Hilfsmittel angewiesen. Die geschriebene Sprache braucht Blatt und Stift, Computer oder Ähnliches.

- Die gesprochene Sprache stellt ein Lautkontinuum dar und erstreckt sich in der Zeit. Das Ohr nimmt die Klänge nicht als Segmente wahr, sondern als Abfolge. Beispielsweise hat der Satz: „Heute Morgen bin ich um halb 8 aufgestanden“ eine zeitliche Ausdehnung von 3,5 Sekunden. Die geschriebene Sprache ist in 
diskrete Einheiten zerlegt (Wörter) und hat eine räumliche Ausdehnung. Der gleiche Satz hat auf einen Blatt die räumliche Ausdehnung von $12 \mathrm{~cm}$ Schrift.

- Die gesprochene Sprache ist dialogisch, die geschriebene monologisch. Das gilt jedoch ebenfalls nur in der Tendenz. Es gibt mündliche Äußerungen, die monologisch sind, wie beispielsweise Vorträge oder Predigten und es gibt Geschriebenes, das dialogisch ist, wie Briefe oder Chats.

\subsection{Die Untersuchung einer mündlichen und schriftlichen Sprache von einem Schüler}

\subsubsection{Also als erstes braucht man für "Antolin" das Internet}

- Globale Planung ist die Einführung in das Thema.

- Gefühle treten nicht auf.

- Beurteilung des Geschehens treten nicht auf.

- Inkohärenz gibt es nicht.

- Referenzmarkierung kommt mit der Einführung eines Referenten vor. In diesem Satz ist 'man' das Indefinitpronomen, 'für Antolin' die Präpositionalphrase, 'das Internet' lexikalische Nominalphrase mit definitem Artikel.

- Kasus- /Genusfehler gibt es nicht.

- Klammerkonstruktion tritt nicht auf.

- Ellipse kommt nicht vor.

- Regiertheit kommt nicht vor.

- V1 gibt es nicht.

- Anbindung gibt es nicht.

- Vorzeitigkeit in der Vergangenheit gibt es nicht.

- 'Also' tritt als Diskuspartikel im Satz auf. 'Als erstes' tritt als Fokuspartikel auf.

- Deixis ohne konkretes Bezugsobjekt gibt es nicht.

\subsubsection{Fürs Antolin muss man als Erstes Internet haben (Schriftsprache)}

- Globale Planung ist die Einführung in das Thema.

- Gefühle treten nicht auf.

- Beurteilung des Geschehens treten nicht auf.

- Inkohærenz gibt es nicht. 
- Refernzmarkierung kommt mit der Einführung eines Referenten vor. In diesem Satz 'für Antolin' die Præpositionalphrase mit definitem Artikel, 'mann' die Indefinitpronomen, 'internet' lexikalische Nominalphrase.

- Kasus- /Genusfehler gibt es nicht.

- Klammerkonstruktion tritt mit dem Modalverb auf (muss ... haben= Modalverb.... V2).

- Ellipse kommt nicht vor.

- Regiertheit kommt nicht vor.

- V1 gibt es nicht.

- Anbindung gibt es nicht.

- Vorzeitigkeit in der Vergangenheit gibt es nicht.

- 'als Erstes' tritt als Fokuspartikel auf.

- 'fürs Antolin' (für das Antolin) ist reduzierte Form.

- Deixis ohne konkretes Bezugsobjekt gibt es nicht.

\subsubsection{Die Rechtschreibung vom Satz " $b$ "}

Die Indefinitpronomen 'mann' muss man nur mit ein " $n$ " schreiben. In 'als Erstes' muss man "E" kleinschreiben. In 'internet' muss man " $i$ " großschreiben, weil Internet ein Substantiv ist. 'Fürs' Antolin ist syntaktisch nicht gut.

\subsubsection{Und dann musst du bei "Antolin" reingehen}

- Globale Planung ist die .

- Gefühle treten nicht auf.

- Beurteilung des Geschehens treten nicht auf.

- Inkohærenz gibt es. Die Person bzw. Pronomen ændert sich. Wæhrend er 'man' benutzt, fængt er an, 'du' zu benutzen.

- Refernzmarkierung ist Beibehaltung eines Referenten mit dem Personalpronomen 'du' und die Beibehaltung eines Referenten mit der Præpositionalphrase 'bei Antolin'. Die Bedeutung mit 'du' ist die Verallgemeinerung statt des Indefinitpronomen 'man'.

- Kasus- /Genusfehler gibt es nicht.

- Klammerkonstruktion tritt mit dem Modalverb auf (muss ... reingehen= Modalverb.... V2). 
- Ellipse kommt nicht vor.

- Regiertheit kommt nicht vor.

- V1 gibt es nicht.

- 'und' und 'dann' sind die Anbindung.

- Vorzeitigkeit in der Vergangenheit gibt es nicht.

- Partikeln gibt es nicht.

- Als reduzierte Form gibt es 'muss' statt der Flexion vom Verb nach 2. Person Singular "musst".

- 'dann' ist Deixis ohne konkretes Bezugsobjekt, weil nichts vor 'dann' erzählt wird.

\subsubsection{Also das heisst "www.antolin" und noch was anderes dazu "de".}

- Globale Planung ist die Hintergrundinformation.

- Gefühle treten nicht auf.

- Beurteilung des Geschehens treten nicht auf.

- Inkohärenz gibt es nicht.

- Refernzmarkierung kommt mit der Einführung eines Referenten vor. ?????

- Kasus- /Genusfehler gibt es nicht.

- Klammerkonstruktion tritt nicht auf.

- Ellipse gibt es im Satz. Das Teil des Prædikats "gehören” fehlt im Satz (was anderes gehört dazu "de").

- Regiertheit kommt nicht vor.

- V1 gibt es nicht.

- 'und' und 'also' sind die Anbindung.

- Vorzeitigkeit in der Vergangenheit gibt es nicht.

- 'also' tritt als Diskuspartikel auf.

- Reduzierte Formen gibt es nicht.

- Deixis ohne konkretes Bezugsobjekt gibt es nicht.

\subsubsection{Dann muss man wissen, wie die Seite heißt (Schriftsprache)}

- Globale Planung ist die Hintergrundinformation. Das Vorwissen ist erforderlich.

- Gefühle treten nicht auf. 
- Beurteilung des Geschehens treten nicht auf.

- Inkohärenz gibt es nicht.

- Referenzmarkierung ist Beibehaltung eines Referenten mit den Indefinitpronomen, 'man' und die Einführung mit der lexikalischen Nominalphrase mit definitem Artikel 'die Seite'.

- Kasus- /Genusfehler gibt es nicht.

- Klammerkonstruktion tritt mit dem Modalverb auf (muss ... wissen= Modalverb.... V2). 'wie die Seite heißt' ist das Nachfeld.

- Ellipse kommt nicht vor.

- Regiertheit kommt nicht vor.

- V1 gibt es nicht.

- 'dann' und 'wie' sind die Anbindung.

- Vorzeitigkeit in der Vergangenheit gibt es nicht.

- Partikeln gibt es nicht.

- Reduzierte Formen gibt es nicht.

- 'dann' ist Deixis ohne konkretes Bezugsobjekt.

\subsubsection{Die Rechtschreibung vom Satz "e"}

Die Indefinitpronomen 'mann' muss man nur mit ein " $n$ " schreiben. In dem Verb 'heist' muss man mit “ $\beta$ ” schreiben. Vor der Konjunktion 'dann' und dem eingeleiteten Nebensatz 'wie' muss man das Komma setzen und am Ende des Satzes muss man den Punkt setzen (nach dem Verb 'heißen').

\subsubsection{Dann wenn du drinne, kommt die Seite}

- Globale Planung ist die Komplikation.

- Gefühle treten nicht auf.

- Beurteilung des Geschehens treten nicht auf.

- Inkohärenz gibt es nicht.

- Referenzmarkierung kommt mit der Einführung eines Referenten mit der lexikalischen Nominalphrase mit definitem Artikel 'die Seite' und mit der Beibehaltung eines Referenten mit dem Personalpronomen 'du'.

- Kasus- /Genusfehler gibt es nicht. 
- Klammerkonstruktion gibt es nicht.

- Ellipse kommt nicht vor.

- Regiertheit kommt nicht vor.

- V1 gibt es nicht.

- 'und', 'dann' und 'subordinierende Konjunktion wenn' sind die Anbindung.

- Vorzeitigkeit in der Vergangenheit gibt es nicht.

- Partikeln gibt es nicht.

- 'drinne' (drinnen) ist die reduzierte Form. 'drinne' ist umgangssprachlich.

- 'dann' ist Deixis ohne konkretes Bezugsobjekt.

\subsubsection{Dann musst du „ersmal“ dein Passwort reineingeben}

- Globale Planung ist die Komplikation.

- Gefühle treten nicht auf.

- Beurteilung des Geschehens treten nicht auf.

- Inkohärenz gibt es nicht.

- Referenzmarkierung ist Beibehaltung eines Referenten mit dem Personalpronomen 'du' und die Einführung eines Referenten mit der lexikalischen Nominalphrase mit dem Possessivpronomen 'dein'.

- Kasus- /Genusfehler gibt es nicht.

- Klammerkonstruktion tritt mit dem Modalverb auf (muss ... reineingeben= Modalverb.... V2).

- Ellipse kommt nicht vor.

- Regiertheit kommt nicht vor.

- V1 gibt es nicht.

- 'dann' ist die Anbindung.

- Vorzeitigkeit in der Vergangenheit gibt es nicht.

- Partikeln gibt es nicht.

- 'ersmal' (erstmal) ist die reduzierte Form.

- 'dann' ist Deixis ohne konkretes Bezugsobjekt.

- 'reineingeben' ist der lexikalische Fehler. 
2.2.10. Wenn man drin ist brauch mann die Kot nummer die du von der Lehrerin gekriegt hast (Schriftsprache)

- Globale Planung ist die Hintergrundinformation.

- Gefühle treten nicht auf.

- Beurteilung des Geschehens treten nicht auf.

- Inkohärenz gibt es nicht.

- Referenzmarkierung ist Beibehaltung eines Referenten mit dem Indefinitpronomen, 'man', der Personalpronomen 'du' und die Einführung eines Referenten mit der lexikalischen Nominalphrase mit definitem Artikel 'die Kot Nummer', der Präpositionalphrase mit definitem Artikel 'von der Lehrerin' und dem Relativsatz 'die'

- Kasus- /Genusfehler gibt es nicht.

- Klammerkonstruktion gibt es im Relativsatz (Relativpronomen und Perfektsatz= die ... gekriegt hast).

- Ellipse kommt nicht vor.

- Regiertheit kommt nicht vor.

- 'wenn' (subordinierende Konjunktion) ist die Anbindung.

- Vorzeitigkeit in der Vergangenheit tritt auf (wen man drin ist, brauch man... gekriegt hast).

- Partikeln gibt es nicht.

- Als reduzierte Form gibt es 'brauch' statt der Flexion vom Verb nach dem Indefinitpronomen "man" 3. Person Singular "braucht".

- Der lexikalische Fehler ist die Verwechselung (Codenummer - Kot nummer).

\subsubsection{Die Rechtschreibung vom Satz "g"}

Die Indefinitpronomen 'mann' muss man nur mit ein " $n$ " schreiben. Die subordinierende Konjunktion 'wen' muss man mit zwei " $n$ " schreiben und am Anfang des Satzes muss man großschreiben (Wenn). Da der Nebensatz durch eine Konjunktion mit einer anderen Konstituente verbunden, muss ein Komma zwischen dem Nebensatz und übergeordnetem Teilsatz stehen (wenn... ist, braucht...). Das Komma vor dem Relativsatz muss man setzen (Zuschreibung einer Eigenschaft zum Referenten des Kopfsubstantivs). Am Ende des Satzes muss man den Punkt setzen.

\section{Vergleich Schriftsprache - gesprochene Sprache des Schülers}

Insgesamt ist Schriftteil kürzer als gesprochene Sprache. Sowohl in der Schriftsprache als auch in der gesprochenen Sprache gibt es keine Fehler bei Kasus, 
Genus und Regiertheit. Inkohärenz tritt nur in der gesprochenen Sprache auf. In der geschriebenen und gesprochenen Sprache sind die Nominalphrase ausgebauter und häufig die Indefinitpronomen "man". In den beiden Sprachen gibt es durchgängige Verwendung des Präsens. In der Schriftsprache treten die Partikeln manchmal, sondern in der gesprochenen Sprache sind die Partikeln häufig.

Reduzierte Formen kommen sowohl in der geschriebenen als auch in der gesprochenen Sprache vor. Aber reduzierte Formen in der Schriftsprache zu benutzen ist nicht gewöhnlich. Ellipse treten in der gesprochenen Sprache auf. Kein Deixis ohne Konkretes Bezugsobjekt gibt es in der Schriftsprache, aber Deixis ohne konkretes Bezugsobjekt wird viel in dem Gesprochenenteil benutzt. Vorzeitigkeit in der Vergangenheit tritt nur im Schriftteil auf. Lexikalische Fehler gibt es in den beiden Sprachen.

\section{Literatur}

Duden, Die Grammatik; Dudenverlag, 2005. S:61

Duden, Die Grammatik; Dudenverlag, 2005. S:62

Dürscheid, Christa (2004). Einführung in die Schriftlinguistik. Wiesbaden.

Fiehler, Reinhard, Bardden, Birgit, Eltermann, Mechthild und Kraft, Barbara (2004). Eigenschaften gesprochener Sprache. Tübingen.

Glück, Helmut (Hg.) (2000). Metzler Lexikon Sprache. Stuttgart.

Glück, Helmut (1987). Schrift und Schriftlichkeit. Eine sprach- und kulturwissenschaftliche Studie. Stuttgart.

Schwitalla, Johannes (22003). Gesprochenes Deutsch. Eine Einführung. (Reihe: Grundlagen der Germanistik, 33). Berlin.

Rath, Rainer (1979). Kommunikationspraxis. Analysen zur Textbildung und Textgliederung im gesprochenen Deutsch. Göttingen.

http://www.isk.rwthaachen.de/uploads/media/gesprochene_vs_geschriebene_Sprache.pdf (Abgerufen am: 08.01.2011) 